\title{
An Adaptive Denoising Method using Empirical Wavelet Transform
}

\author{
Anjana Francis \\ Student \\ Nehru college of Engineering and Research Centre
}

\begin{abstract}
Empirical Wavelet Transform is a new adaptive signal decomposition technique. In signal processing, adaptive representation of signal is very important. This is very useful for denoising, decompression etc. This paper presents an adaptive denoising technique using Empirical wavelet transform. Experiments presented showing the effectiveness of this method based on their signal to noise ratio.
\end{abstract}

\section{General Terms}

Empirical Wavelet Transform, Adaptive, Signal to Noise Ratio, Time-Frequency Analysis.

\section{Keywords}

AM-FM Components, TF Representation, EWT.

\section{INTRODUCTION}

In signal processing, time frequency analysis means analyze a signal in both time and frequency domain simultaneously. Signal analysis in adaptive manner is very useful for signal processing. Generally we can represent a signal as a linear combination of basis functions. In methods like wavelet and Fourier transform these basis functions are derived independently, but in adaptive techniques these functions are derived from the information contained in the signal. Jerome Gilles proposed a new approach to build adaptive wavelets that is known as Empirical Wavelet Transform. This method is able to separate the Nonlinear and Non-stationary part of the signal. These components have a compact support Fourier spectrum.

These techniques are applicable for signal denoising. Denoising is a technique that is used to remove noise content from the signal and to reconstruct the original signal. In the field of signal processing denoising is still a challenging problem. So many methods are there to remove noise content from the signal and to recover the original signal. Each of these methods has their own advantages and limitations. Wavelet transform analysis has been widely used for the purpose of denoising. Traditional denoising schemes are based on linear methods. That is not suitable for nonlinear and non-stationary signals. To perform signal denoising in nonlinear and non-stationary signals an adaptive signal denoising method using Empirical wavelet transform is proposed in this paper

\section{EMPIRICAL WAVELET}

\section{TRANSFORM}

\subsection{Empirical Wavelet}

It is a type of wavelet that is adapted to the processed signal. The construction of this wavelet is equivalent to the construction of Band-pass filters. Empirical wavelets provide adaptability to the signals. Using this wavelet we can separate a given signal as a number of modes known as Amplitude Modulated-Frequency Modulated components that is AM-FM components. This AM-FM components have a compactly

\author{
Muruganantham C \\ Assistant professor \\ Nehru college of Engineering and Research Centre
}

supported Fourier spectrum. Here, segmentation of Different modes is equivalent to the segmentation of Fourier spectrum.

Assume that the Fourier spectrum is divided into N segments. There is a limit between each segment $\omega_{\mathrm{n}}$. Segmentation of the spectrum is an important task, because, this segmentation provides adaptability. Our aim is to separate different portions of the spectrum that corresponds to different modes. In order to divide the spectrum into $\mathrm{N}$ segments, we need a total of $\mathrm{N}+1$ boundaries, but the limit of Fourier spectrum is in between 0 and $\pi$ we need a total of n- 1 extra boundaries. To find such boundaries first detect the local maxima in the spectrum and arrange them in the decreasing order. Assume that the algorithm found $\mathrm{M}$ maxima, two cases can appear:[1]

$\mathrm{M} \geq \mathrm{N}$ : The algorithm found enough maxima to define the required number of segments, then we keep only the first $\mathrm{N}-1$ maxima.

$\mathrm{M}<\mathrm{N}$ : The signal has less modes than expected, then we keep all the detected maxima than expected, then we keep all the detected maxima and reset $\mathrm{N}$ to appropriate value.

With this set of maxima plus 0 and $\pi$ we define the boundaries $\omega_{\mathrm{n}}$ of each segment. This is the centre between two consecutive maxima. $\left(\omega_{0}=0\right.$ and $\left.\omega_{\mathrm{n}}=\pi\right)$.

The Empirical scaling function and Empirical wavelet can be expressed as follows.[1]

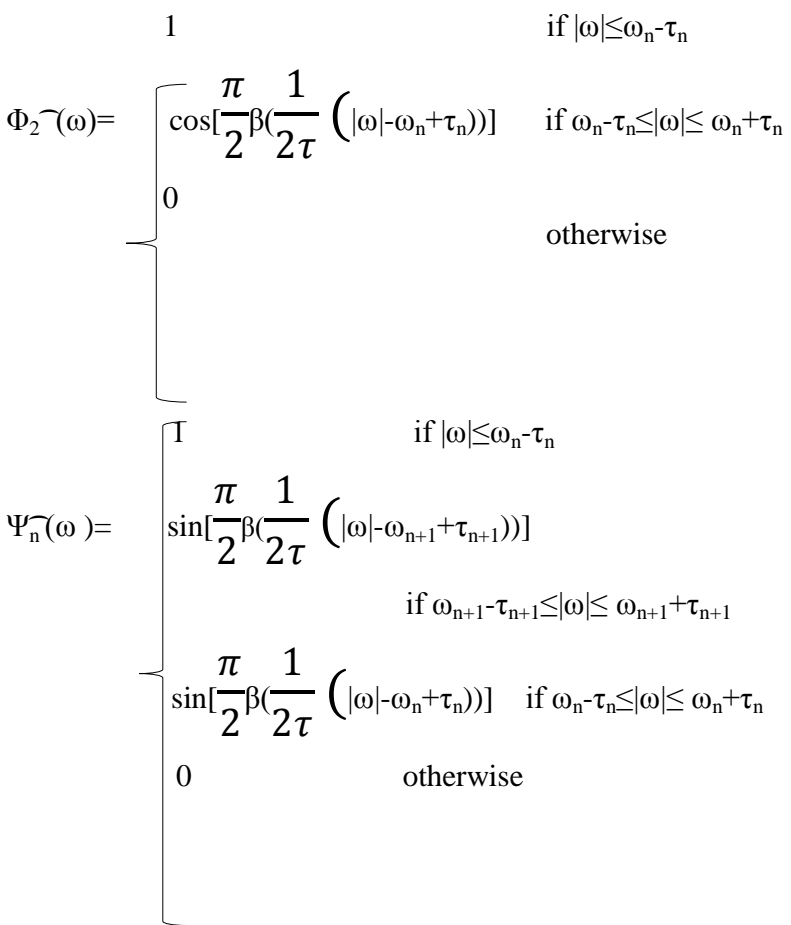

\subsection{Empirical Wavelet Transform}

Empirical wavelet transform can be defined in the same way as that of classical wavelet transform. 
The detail coefficients are given by the inner product with the Empirical wavelets[1]

$\left.\mathrm{W}_{\mathrm{f}}^{\mathrm{e}}(\mathrm{n}, \mathrm{t})=\left\langle\mathrm{f}, \psi_{\mathrm{n}}\right\rangle=\int \mathrm{f}(\tau) \overline{\psi_{\mathrm{n}}(\tau-\mathrm{t}}\right) \mathrm{d} \tau=\left(\widehat{\mathrm{f}}(\omega) \overline{\psi_{\mathrm{n}}(\omega)}\right)^{\mathrm{v}}$

And the approximation coefficients by the inner product with the scaling function[1]

$\left.\left.\mathrm{W}_{\mathrm{f}}^{\varepsilon}(0, \mathrm{t})=\left\langle\mathrm{f}, \varphi_{1}\right\rangle=\int \mathrm{f}(\tau) \overline{\varphi_{1}(\tau-\mathrm{t}}\right) \mathrm{d} \tau=\left(\widehat{\mathrm{f}}(\omega) \overline{\varphi_{1}(\omega}\right)\right)^{\mathrm{v}}$

Where $\psi_{\mathrm{n}}(\omega)$ and $\varphi_{1}(\omega)$ are defined by

$$
\begin{gathered}
\Phi_{\mathrm{n}}^{-}(\omega)= \begin{cases}1 & \text { if }|\omega| \leq(1-\gamma) \omega_{\mathrm{n}} \\
\cos \left[\frac{\pi}{2} \beta\left(\frac{1}{2 \gamma}(|\omega|-(1-\gamma))\right]\right. & \text { if }(1-\gamma) \omega_{\mathrm{n}} \leq|\omega| \leq(1+\gamma) \omega_{\mathrm{n}} \\
0 & \text { otherwise }\end{cases} \\
\Psi_{\mathrm{n}}^{-}(\omega)= \begin{cases}1 & \text { if }(1+\gamma) \omega_{\mathrm{n}} \leq|\omega| \leq(1-\gamma) \omega_{\mathrm{n}+1} \\
\cos \left[\frac{\pi}{2} \beta\left(\frac{1}{2 \gamma}\left(|\omega|-(1-\gamma) \omega_{\mathrm{n}+1}\right)\right)\right] & \text { if }(1-\gamma) \omega_{\mathrm{n}+1} \leq|\omega| \leq(1+\gamma) \omega_{\mathrm{n}+1} \\
\sin \left[\frac{\pi}{2} \beta\left(\frac{1}{2 \gamma}\left(|\omega|-(1-\gamma) \omega_{\mathrm{n}}\right)\right)\right] & \text { if }(1-\gamma) \omega_{\mathrm{n}}-\tau_{\mathrm{n}} \leq|\omega| \leq(1+\gamma) \omega_{\mathrm{n}} \\
& \text { otherwise }\end{cases}
\end{gathered}
$$

The reconstruction is obtained by,

$\mathrm{f}(\mathrm{t})=\mathrm{W}_{\mathrm{f}}^{e}(0, \mathrm{t}) * \varphi_{1}(\mathrm{t})+\sum^{\mathrm{N}}{ }_{\mathrm{n}=1} \mathrm{~W}_{\mathrm{f}}^{e}(\mathrm{n}, \mathrm{t}) * \psi_{\mathrm{n}}(\mathrm{t})$

$=\left(\widehat{\mathrm{W}_{\mathrm{f}}}(0, \omega) \widehat{\varphi_{1}}(\omega)+\sum_{\mathrm{n}=1}^{\mathrm{N}} \mathrm{W}_{\mathrm{f}}^{\varepsilon}(\mathrm{n}, \omega) \psi_{\mathrm{n}}-(\omega)\right)$

\section{ALGORITHM FOR EWT}

Step 1: Take an ECG signal.

Step 2: Apply some Noise and consider this as the input signal.

Step 3: Find spectrum of that signal by applying Fourier Transform.

Step 3: Find out all the local maxima's.

Step 4: Find out all mid points between adjacent local maxima's.

Step 4: Apply window function. Multiplies these midpoints with this window function.

Step 5: Take inverse Fourier transform.

\section{EXPERIMENTAL RESULTS}

Table 1. SNR Values for Different Iterations

\begin{tabular}{|l|l|l|}
\hline $\begin{array}{l}\text { ITERATION } \\
\text { NUMBER }\end{array}$ & $\begin{array}{l}\text { SNR BEFORE } \\
\text { DENOISING }\end{array}$ & $\begin{array}{l}\text { SNR AFTER } \\
\text { DENOISING }\end{array}$ \\
\hline 1 & 19.8681 & 26.7961 \\
\hline
\end{tabular}

\begin{tabular}{|l|l|l|}
\hline 2 & 16.8578 & 25.3499 \\
\hline 3 & 15.0969 & 24.0939 \\
\hline 4 & 13.8475 & 23.1047 \\
\hline 5 & 12.8784 & 22.3402 \\
\hline
\end{tabular}

The above table shows the Signal to Noise Ratio values for an ECG signal for different iterations. Signal to Noise Ratio values are calculated before and after iteration. For analysis five iterations are used. From the table it is clear that the Signal to Noise Ratio values are higher for Empirical wavelet transform method. Fig 1 shows a graph that plotted the SNR before and after iteration.

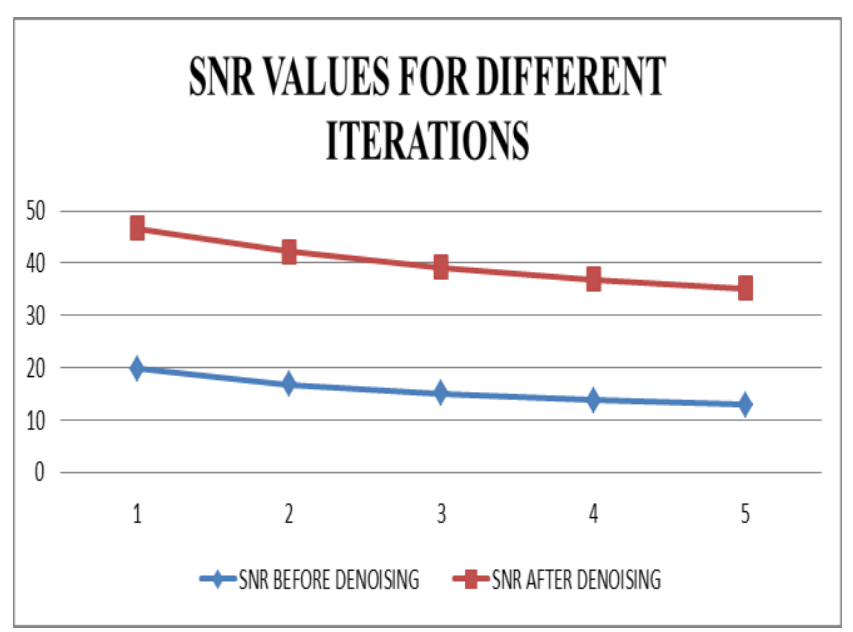

Fig 1. SNR value comparison Before and After Denoising

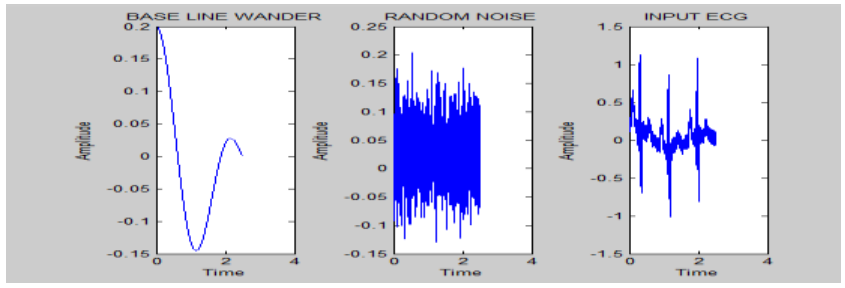

Fig 2. Input Noisy ECG signal

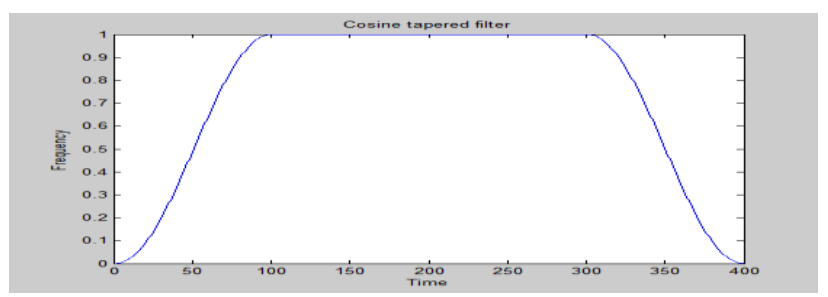

Fig 3. Cosine Tapered Filter

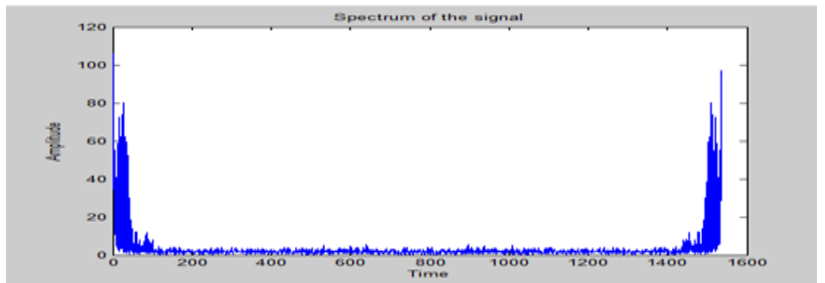

Fig 4. Spectrum of the Signal 


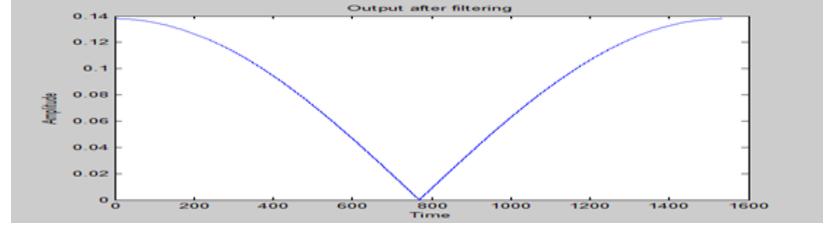

Fig 5a

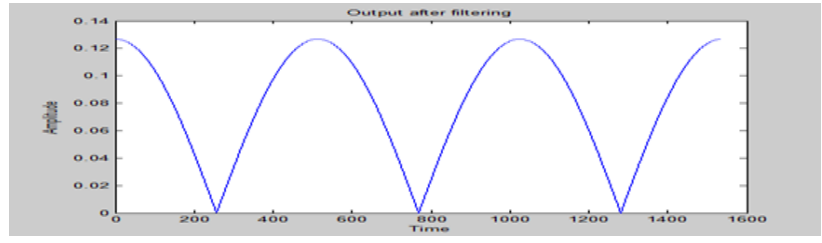

Fig 5b

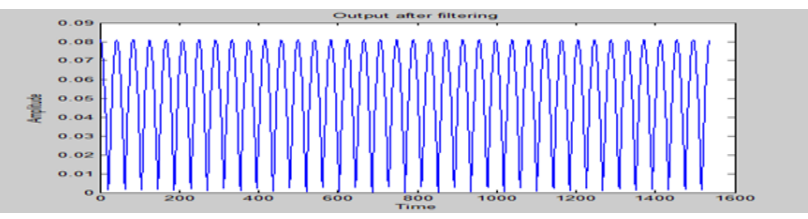

Fig 5c

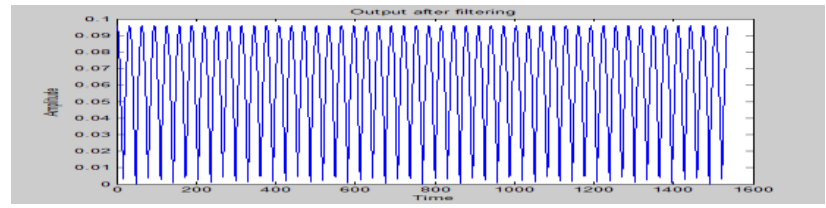

Fig 5d

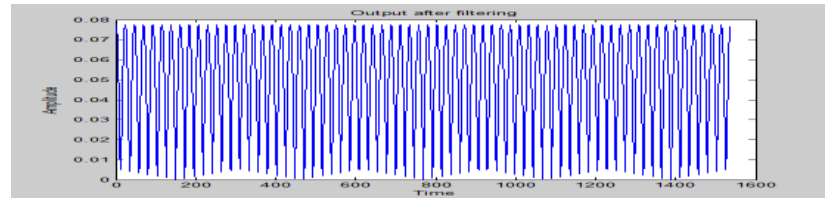

Fig $5 e$

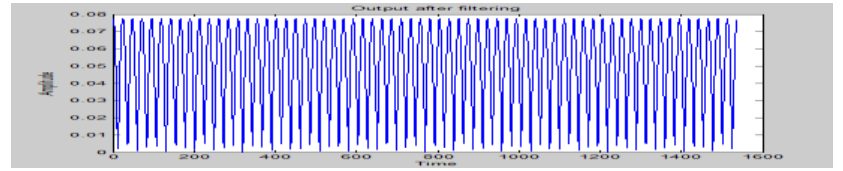

Fig $5 f$

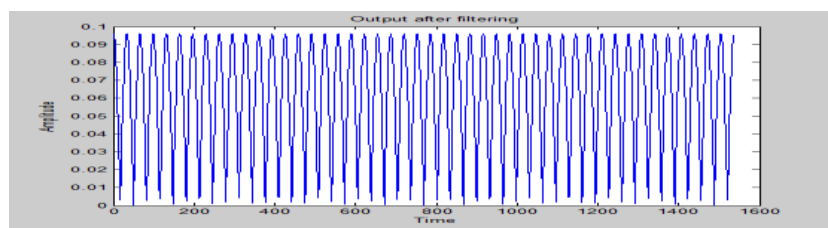

Fig $5 g$

Fig 5(a-g). Modes Extracted by EWT

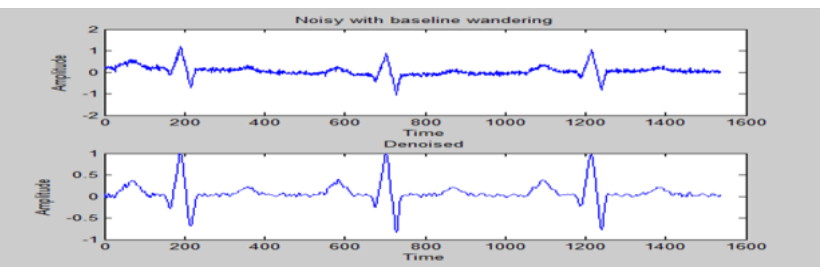

Fig 6. Noisy Signal with Baseline Wandering and Denoised signal using EWT
Fig 2-6 shows output of different stages involved in the empirical wavelet transform Decompsition process. Fig 2 shows the noisy ECG signal. This noisy signal consists of two types of noises, baseline wander noise and powerline interference. Here we multiply the spectrum of the signal with a cosine tapered filter, that is shown in figure 3. Fig 5 shows difeerent modes extracted using EWT. Fig 6 is the denoised signal after applying the EWT.

\section{CONCLUSION}

Experiments were performed on ECG signal. ECG signal is one of the nonlinear biological signal. This ECG signal contains baseline wandernoise and random noise. Table 1 shows the SNR values obtained Before and after Denoising. Denoising using EWT gives better results. In future we can extend this concept to images also. The procedure used for 1D signal is also applicable for 2D signals. This method is self adaptive and using this method it is able to seperate nonlinear and non-stationary parts of the signals. In future this method can be used for deconvolution. Here IMFs are not orthogonal, to make it orthogonal different orthogonalisation procedures can be used.

\section{ACKNOWLEDGMENTS}

The authors would like to express their sincere gratitude towards the authorities of the Department of Electronics and Communication Engineering, Nehru College of Engineering and Research Centre, for providing constant support throughout this work.

\section{REFERENCES}

[1] Jerome Gilles, Empirical wavelet transform, IEEE trans. On signal processing, vol. Xx, no. Xx, February 2013.

[2] Ingrid Daubechies, Jianfeng Lu, Hau-Tieng Wu, Synchrosqueezed Wavelet Transforms: An Empirical Mode Decomposition-like Tool.

[3] Patrick Flandrin, Empirical Mode Decomposition as a Filter Bank, IEEE signal processing letters, vol. 11, no. 2, february 2004 .

[4] Sreedevi Gandham, T. Sreenivasulu Reddy, Enhanced Signal Denoising Performance by EMD-based Techniques.

[5] Md. Ashfanoor Kabir \& Celia Shahnaz, Comparison of ecg signal denoising algorithms in emd and wavelet domains.

[6] P. Trnka, M. Hofreiter, The Empirical Mode Decomposition in Real-Time.

[7] Norden E. Huang1,Steven R. Long, Samuel S. P. Shen and Jin E. Zhang, Applications of Hilbert-Huang transform to non-stationary financial time series analysis.

[8] Maria E. Torres , Marcelo A. Colominas , Gaston Schlotthauer, Patrick Flandrin, A complete ensemble empirical mode decomposition with adaptive noise.

[9] Yannis Kopsinis, Stephen (Steve) McLaughlin, Development of EMD-based denoising methods inspired by Wavelet thresholding.

[10] Sonam Maheshwari, Dimpy, Application of Empirical Mode Decomposition in Denoising a Speech Signal. 